\title{
Actual Dose-Reduction Strategies in Cardiac Computed Tomography
}

\author{
Daniel Cernica', Roxana Hodas¹, Elisabeta Himcinschi¹, Elena Beganu¹, Theodora Benedek1,2 \\ 1 Center of Advanced Research in Multimodality Cardiac Imaging, Cardio Med Medical Center, Tîrgu Mureș, Romania \\ 2 University of Medicine and Pharmacy, Tîrgu Mureș, Romania
}

\section{CORRESPONDENCE}

\section{Roxana Hodas}

Str. 22 Decembrie 1989 nr. 76

540124 Tîrgu Mureș, Romania

Tel: +40 265217333

E-mail: roxana.hodas@yahoo.ro

\section{ARTICLE HISTORY}

Received: June 7, 2017

Accepted: July 25, 2017
Daniel Cernica • Str. 22 Decembrie 1989 nr. 76 , 540124 Tîrgu Mureș, Romania. Tel: +40 265217333. E-mail: daniel.cernica@gmail.com

Elisabeta Himcinschi • Str. 22 Decembrie 1989 nr. 76 540124 Tîrgu Mureș, Romania. Tel: +40 265217333. E-mail: eli_himcinschi@yahoo.com

Elena Beganu • Str. 22 Decembrie 1989 nr. 76 540124 Tîrgu Mureș, Romania. Tel: +40 265217333. E-mail: beganu.elena@yahoo.com

Theodora Benedek • Str. Gheorghe Marinescu nr. 38 540139 Tîrgu Mureș, Romania. Tel: +40 265215551.

E-mail: theodora.benedek@gmail.com

\begin{abstract}
Computed tomography (CT) in cardiac examination is a powerful imaging tool that has developed rapidly during the last decade and continues to increase its potential by bringing novel technologies. Due to its noninvasive character, cardiac CT became a largely used method in detecting coronary diseases or functional issues at the expense of conventional coronary angiography. The accuracy of images has also increased, especially since new generation dual-source multi-slice detectors were developed. Although there are continuous improvements that serve to gain better-quality images, thus increasing their diagnostic accuracy, there is an inconvenient that became a serious topic for debate in the current literature: exposure to higher doses of radiation during cardiac CT examinations. Fortunately, physicians and manufacturers are taking into consideration the need to apply new strategies for radiation dose-reduction. Thus, this objective can be achieved by using patient-tailored dose-reduction strategies and by modulating the technical features of the CT scanners in order to gather highquality images with minimal radiation exposure. The aim of this manuscript was to review the current literature data on dose-reduction strategies that are used for cardiovascular computed tomography scans.
\end{abstract}

Keywords: dose-reduction strategy, cardiac computed tomography

\section{INTRODUCTION}

Cardiac computed tomography (CT) is a very promising modern imaging technique, which has abruptly emerged over the last decade. ${ }^{1}$ Nowadays, it is known that this noninvasive technique has a high grade of accuracy, especially since manufacturers have developed 64- or more slices CT systems. Since then, the importance of this reliable technique has increased in indentifying coronary artery disease, and it is widely accepted at the moment as a powerful diagnostic tool, an alternative to conventional coronary angiography. Despite its great diagnostic value, physicians have raised important concerns over the adverse side of cardiac computed tomography: radiations. With continuous technical advances, CT scans play an important role in revealing and detailing atherosclerotic plaques on coronary arteries. Alongside those significant improvements, multi-slice CT (MSCT) bears a considerable volume of radia- 
tions. ${ }^{2-5} \mathrm{~A}$ radiation dose of approximately $40 \mathrm{mSv}$ is delivered by a cardiac MSCT scan during a routine examination without any dose-saving protocols. ${ }^{6}$ Due to the increased importance of the method and its high utilization, its long-term side effects and especially malignant consequences have become a serious topic of debate in the current literature. ${ }^{7-9}$

Understanding how the amount of radiations influences the appearance of secondary disorders and developing strategies for lowering the emission of radiations, are the actual challenging milestones. The exposure to radiation is dependent on many factors related to CT scanners, such as scanner geometry, electrocardiogramgating (ECG-gating) applications, tube voltage levels, tube current levels, slice thickness, and pitch value, and most of these parameters are controlled by CT operators during procedures, according to the desired image quality. Therefore, it is necessary to develop new scanning and acquisition strategies in order to lower the exposure to radiation, without compromising the quality of the images. ${ }^{10}$

This clinical review discusses potential dose-reduction methods available at the moment for cardiovascular computed tomography scanning.

At present, there are several types of strategies available for decreasing exposure to radiations delivered during a routine cardiac $\mathrm{CT}$, with the condition to preserve image quality. These dose-reduction methods are based on the modulation of various parameters of the CT scanner, depending on either the experience of the radiologist or on the scanner manufacturer settings. Hence, it is important to assess when is it possible to use an appropriate strategy to lower radiation exposure and to maintain the radiation dose as low as reasonably achievable (ALARA). ${ }^{11}$

Current modalities for dose reduction consist in balancing several machine-related factors such as scan range and geometry of scanning, post-processing filters, the tube current emitted and the voltage of the scanner tube, CT pitch, slice thickness and shielding, as well as ECGgating scan.

\section{POST-PROCESSING FILTERS}

The most effective modality for dose reduction consists in the use of post-processing filters after a scan was performed. There are algorithms analyzing the scanned image that are capable to eliminate image noise but preserve image quality. The amount of dose reduction is estimated to be up to $80 \%$, and usually these filters are applied to images acquired using low-dose radiation protocols. ${ }^{12}$

\section{ECG-GATED SCANNING - PROSPECTIVE ACQUISITION}

ECG-gated scanning represents another modality for successfully lowering the exposure, by performing a CT scan based on heart rate and the phases of the cardiac cycle. An efficient approach is represented by the prospective ECGtriggered acquisition, defined by recording of data during a determinate phase of the cardiac cycle triggered by the QRS signal, which will activate the gantry rotation while the table is fixed. This technique is repeated until the entire anatomical area of interest is covered. The benefit of this technique in terms of radiation exposure is a reduction in dose of an estimated $68 \%$, without compromising the quality of the recorded images. The requirement to obtain a satisfactory result is a regular and low heart rate, because the QRS signal and the R-R interval are calculated in order to trigger the scanning process. Although there are enormous benefits associated with this method, it is used only to analyze anatomical areas, because the functional data is not delivered due to the short acquisition during a predefined period of the R-R interval. ${ }^{13,14}$

\section{ECG-GATED SCANNING - PROSPECTIVE ACQUISITION AND HIGH PITCH}

Another dose reduction strategy includes the prospective ECG-gated scan associated with a large pitch of up to 3.4 on second-generation dual-source CT scanners. The higher the value of pitch level, the lower the amount of radiation will be. Hence, image acquisition is made by rotating the gantry at larger lengths, without a lack of images due to the presence of gaps between successively recorded data, such as in past-generation CT scanners. The method is recommended in patients with a low heart rate and a stable rhythm and can also decrease the amount of emitted radiations that could reach an estimated value of $1 \mathrm{mSv}$ per examination. ${ }^{15,16}$

\section{ECG-GATED SCANNING - RETROSPECTIVE ACQUISITION}

Retrospective ECG-gated acquisition with a low pitch value is a frequently used mode for cardiac CT scans, delivering a high dose of radiation with the benefit of gaining good-quality images for functional aspects of the examined heart. One possible method to decrease the high dose delivered with this method is the modulation of tube current. ${ }^{17}$ Hence, the tube current is set to be at a maximum level during a predefined single phase of the heart 
cycle (mid-to-end of the diastole), resulting in a reduced exposure of up to $50 \%$ due to the overall dose of radiations emitted during a cardiac CT scan. ${ }^{18-20}$ The factors that need to be modulated are the value of the tube current and its duration, which need to be as low as possible during specific phases of the cardiac cycle, and heart rate needs to be regular and lowered in order to increase the recording time up to $75 \%$ of the corresponding cardiac phase. ${ }^{21,22}$

\section{NON-ECG GATED SCANNING}

Another option to gather $\mathrm{CT}$ images using a low radiation dose consists in non-ECG gated acquisitions. However, they can only be used in special cases such as analysis of the aorta, pulmonary veins, or the left atrium. ${ }^{17}$

The next important strategy consists in properly preparing the patient before performing the scan. It includes filters to attenuate low energies delivered by the X-ray tube, which might not be necessary during certain scans and does not contribute to image acquisition, adding only an exceeding amount of radiations to the overall dose. The choice for the size of $\mathrm{X}$-ray filters should be made rationally, considering that the smaller the filter is, the higher the dose-reduction. The radiology engineer has to balance the beneficial aspects by using a small filter and the size of the resulting field of view, which in this case would be smaller. Collimators are filter-like tools, representing another option for protecting the patients from unnecessary high doses during X-rays. They are positioned near the CT tube in order to limit the X-ray stream exposure only in the area of interest. Additional protective measures include the use of bismuth-latex shields, positioned between the X-ray beam and the protected area. It would be essential in the examination of female patients due to the unnecessary exposure of the breasts during a CT scan. It has been shown that this strategy would decrease the exposure up to $57 \%$, but it is actually not recommended during routine scans because of the important degradation of images. Therefore, it would need further improvements in manufacturer technology in order to become usable during routine examinations and to offer high protection on certain anatomical areas, such as the breasts in female patients. ${ }^{18-20}$

\section{TUBE POTENTIAL}

On the other hand, there are a few options related to the setup of the machine, which can be adjusted in order to increase the balance between benefits and side effects during a cardiac CT examination. Another CT scan factor, which can be an effective dose-reduction strategy, is tube poten- tial modulation. Tube potential is defined as the electric potential that gives the tube its energy and radiation intensity.

\section{TUBE CURRENT}

Tube current represents the process of accelerating electrons through the X-ray tube related to the time unit, measured as milliampers per second (mAs). It is proportional with the amount of emitted radiation and inversely proportional with image noise. Thus, by lowering the current of the tube, the radiation dose would decrease, at the expense of image quality. However, regarding cardiovascular imaging, it is possible to use low currents and obtain satisfactory data. To enhance this beneficial feature of a CT scan, radiology engineers can also modulate other factors such as anatomy-based scanning or acquisition-based scanning on ECG-triggered scans. Given that most cardiac CT examinations are performed using retrospective ECG-gated acquisitions, it is crucial to modulate the tube current during the acquisition phase. Using the ECG-gated mode, tube current emission is active during the whole cardiac cycle while being correlated to a single breath-hold. Then, image reconstruction will follow, with the analysis of only one phase of cardiac cycle. Thus, this technique could use less radiation by applying rational adjustments: the current tube would be higher when a specific phase needs to be analyzed, using the high-quality images during the reconstruction stage, and lower dose exposure during other phases of the cardiac cycle that are not of interest, resulting in increased image noise. The amount of dose-reduction by performing a cardiac CT with this strategy is between $30 \%$ to $50 \% .{ }^{22,23} \mathrm{~A}$ similar technique is the anatomy-based tube current adaptation, where the tube current is automatically adjusted to the body shape, analyzed in a threedimensional design. Despite its contribution in reducing the radiation dose with about $20 \%$, currently this method has a relatively low utility due to the small angular fluctuation of the attenuation of the heart. ${ }^{24}$

\section{TUBE VOLTAGE}

Tube energy, known as tube voltage, is the power generated by the X-ray tube during a CT scan. The latest generation scanners have increased the level of delivered energy, making the examination time shorter, alongside with increasing the dose of radiation. The maximum level of the tube energy is $100 \mathrm{~kW}$, which corresponds to an amount of radiations of $100 \mathrm{mGy}{ }^{25}$ Current protocols use a higher power for low-normal weight patients due to lower attenu- 
ation of $\mathrm{x}$-rays in these patients. Despite this, it has been recently shown that there is possibility to gather goodquality images with a decreased tube voltage by increasing iodine absorption when the heart is exposed to a lower voltage. ${ }^{26-28}$

\section{CONCLUSION}

Cardiac computed tomography is reliable imaging method, which has grown rapidly in the last decade and continues to develop in order to achieve what patients and clinicians are looking for - the best decision-making process in terms of diagnosis. However, these improvements also bring side effects, which have to be managed judiciously by clinicians, radiology engineers, and manufacturers. Currently, there are many options to overcome this inconvenient. Dose-reduction strategies should be known by every clinician in order to inform patients on the beneficial part of CT scans, as well as their side effects. Thus, strategies for lowering radiation exposure must begin by analyzing the patients' features, such as the body weight to adapt the amount of beam potential and the heart rate and rhythm, and also information regarding the type of scan functional or anatomical. Cardiac CT scan strategies that are tailored to patient characteristics, are based on anatomical features, and use the latest scanner technologies according to dose-saving strategies should represent the current protocol.

\section{CONFLICT OF INTEREST}

Nothing to declare.

\section{REFERENCES}

1. Taylor AJ, Cerqueira M, Hodgson JM, et al. CCF/SCCT/ACR/AHA ASE/ASNC/NASCI/SCAI/SCMR 2010 appropriate use criteria for cardiac computed tomography. A report of the American College of Cardiology Foundation Appropriate Use Criteria Task Force, the Society of Cardiovascular Computed Tomography, the American College of Radiology, the American Heart Association, the American Society of Echocardiography, the American Society of Nuclear Cardiology, the North American Society for Cardiovascular Imaging, the Society for Cardiovascular Angiography and Interventions, and the Society for Cardiovascular Magnetic Resonance. J Am Coll Cardiol. 2010;56:18641894.

2. Buth J, Disselhoff B, Sommeling C, Stam L. Color-flow duplex criteria for grading stenosis in infrainguinal vein grafts. J Vasc Surg. 1991;14:716-726.

3. Sun $\mathrm{Z}, \mathrm{Ng} \mathrm{KH}$. Diagnostic value of coronary $\mathrm{CT}$ angiography with prospective ECG-gating in the diagnosis of coronary artery disease: a systematic review and meta-analysis. Int J Cardiovasc Imaging. 2012;28:2109-2119.
4. Sun Z, Choo GH, Ng KH. Coronary CT angiography: current status and continuing challenges. Br J Radiol. 2012;85:495-510.

5. Sun Z. Multislice CT angiography in coronary artery disease: Technica developments, radiation dose and diagnostic value. World J Cardiol. 2010;2:333-343.

6. Paul JF, Abada HT. Strategies for reduction of radiation dose in cardiac multislice CT. Eur Radiol. 2007:17:2028.

7. Brenner DJ, Hall EJ. Computed tomography - an increasing source of radiation exposure. N Engl J Med. 2007;357:2277-84

8. Hausleiter J, Meyer T, Hermann F, et al. Estimated radiation dose associated with cardiac CT angiography. JAMA. 2009;301:500-507.

9. Raff GL, Chinnaiyan KM, Share DA, et al. Radiation dose from cardiac computed tomography before and after implementation of radiation dosereduction techniques. JAMA. 2009;301:2340-2348.

10. Halliburton SS, Abbara S, Chen MY, et al. SCCT guidelines on radiation dose and dose-optimization strategies in cardiovascular CT. J Cardiovasc Comput Tomogr. 2011;5:198-224.

11. Sun Z, Ng K H. Multislice CT angiography in cardiac imaging. Part III: radiation risk and dose reduction. Singapore Med J. 2010;51:374-380.

12. De Geer J, Sandborg M, Smedby O, Persson A. The efficacy of 2D, nonlinear noise reduction filtering in cardiac imaging: A pilot study. Acta Radiologica. 2011;52:716-722.

13. Bischoff $\mathrm{B}$, Hein $\mathrm{F}$, Meyer $\mathrm{T}$, et al. Impact of a reduced tube voltage on CT angiography and radiation dose: Results of the PROTECTION I study. JACC Cardiovasc Imaging. 2009;2:940-946.

14. Halliburton SS, Abbara S, Chen MY, et al. SCCT guidelines on radiation dose and dose-optimization strategies in cardiovascular CT. J Cardiovas Comput Tomogr. 2011;5:198-224.

15. Leschka S, Stolzmann P, Desbiolles L, et al. Diagnostic accuracy of highpitch dual-source CT for the assessment of coronary stenosis: First experience. Eur Radiol. 2009;19:2896-2903.

16. Alkadhi H, Stolzmann P, Desbiolles L, et al. Low-dose, 128-slice, dualsource CT coronary angiography: Accuracy and radiation dose of the high-pitch and the step-and-shoot mode. Heart. 2010;96:933-938.

17. Wagner $\mathrm{M}$, Butler $\mathrm{C}$, Rief $\mathrm{M}$, et al. Comparison of non-gated vs. electrocardiogram-gated 64-detector-row computed tomography for integrated electroanatomic mapping in patients undergoing pulmonary vein isolation. Europace. 2010;12:1090-1097.

18. Jakobs TF, Becker CR, Ohnesorge B, et al. Multislice helical CT of the heart with retrospective ECG gating: Reduction of radiation exposure by ECG-controlled tube current modulation. Eur Radiol. 2002;12:1081-1086.

19. Wintersperger B, Jakobs T, Herzog P, et al. Aorto-liac multidetector-row $\mathrm{CT}$ angiography with low $\mathrm{kV}$ settings:improved vessel enhancement and simultaneous reduction of radiation dose. Eur Radiol. 2005:15:334-341.

20. Halliburton SS, Abbara S, Chen MY, et al. SCCT guidelines on radiation dose and dose-optimization strategies in cardiovascular CT. J CardiovasC Comput Tomogr. 2011;5:198-224.

21. Hopper KD, King SH, Lobell ME, et al. The breast: In-plane x-ray protection during diagnostic thoracic $\mathrm{CT}$ - shielding with bismuth radioprotective garments. Radiology. 1997;205:853-858

22. Fricke BL, Donnelly LF, Frush DP, et al. In-plane bismuth breast shields for pediatric CT: Effects on radiation dose and image quality using experimental and clinical data. AJR Am J Roentgenol. 2003;180:407-411.

23. DeFrance T, Dubois E, Gebow D, et al. Helical prospective ECG-gating in cardiac computed tomography: Radiation dose and image quality. Int $J$ Cardiovasc Imaging. 2010;26:99-107.

24. Abada HT, Larchez C, Daoud B, et al. MDCT of the coronary arteries: feasibility of low-dose CT with ECG-pulsed tube current modulation to reduce radiation dose. AJR Am J Roentgenol. 2006;186:S387-S390.

25. Jakobs TF, Becker CR. Ohnesorge B, et al. Multislice helical CT of the heart with retrospective EKG gating: reduction of radiation exposure by ECG-controlled tube current modulation. Eur Radiol. 2002:12:1081-1086.

26. Xu L, Zhang Z. Coronary CT angiography with low radiation dose. Int J Cardiovasc Imaging. 2010;26:17-25.

27. Starck G, Lonn L, Cederblad A,Forssell-Aronsson E, Sjostrom L, Alpsten M. A method to obtain the same levels of CT image noise for patients of various sizes, to minimize radiation dose. Br J Radiol. 2002;75:140-150.

28. Sigal-Cinqualbre AB, Hennequin R, Abada HT, Chen X, Paul JF. Lowkilovoltage multi-detector row chest CT in adults: feasibility and effecton image quality and iodine dose. Radiology. 2004;231:169-174. 